\title{
Shear effects in thickening
}

\author{
S.P. Usher Department of Chemical and Biomolecular Engineering, University of Melbourne, Australia \\ R. Spehar Department of Chemical and Biomolecular Engineering, University of Melbourne, Australia \\ P.J. Scales Department of Chemical and Biomolecular Engineering, University of Melbourne, Australia
}

\begin{abstract}
One-dimensional computational dewatering models which account for the influence of networked particulate bed compression, have enabled accurate predictions of dewatering in sedimentation, centrifugation and filtration processes in both the laboratory and industry. However, in certain processes, such as gravity thickening, the models have been observed to under predict the performance (in terms of solids throughput) by up to a factor of 100 for a given underflow solids concentration. This discrepancy can be credited to shear and channelling effects on the fundamental dewatering material properties which are unaccounted for in current models. The expectation when shear is applied is that local pressure gradients will be produced resulting in the expulsion of water from the aggregates and subsequent densification. This aggregate densification phenomenon has been shown to be very significant for flocculated aggregates in the presence of shear and/or a solid surface. Shear effects can involve raking, flow near sloped walls and also buffeting of aggregates in un-networked fluidised regions of the thickener.
\end{abstract}

Experiments that look to characterise this behaviour have shown that there is an optimum shear rate beyond which a further increase is detrimental to performance. This optimum is a trade off between the densification of aggregates which dominates at low shear rates and the disintegration of aggregates which becomes more significant as shear rate and time of shear increases. Changes to the extent of aggregate densification can be inferred from changes in the material properties with shear and residence time in raked settling tests, Couette-fluidisation tests and pilot thickener operations. Analysis of data from such tests enables the development of more sophisticated dewatering models that can predict the rate of densification as a function of the local shear rate. This type of analysis has the potential to identify whether aspects of full scale thickener design and operation actually enhance or diminish dewatering performance.

\section{Introduction}

Dewatering processes that are industrially employed to increase the solids concentrations of particulate suspensions include gravity thickening, sedimentation/consolidation in large tailing impoundments, centrifugation and filtration processes.

Figure 1 shows typical solids concentration ranges for the outputs of each of these processes overlayed on a typical compressive yield stress curve (see 1.1) which gives an indication of the network strength of the particulate suspension.

From

Figure 1, filtration is identified as the process that can achieve the highest output solids concentration, but is often a batch process with limited throughput and very high maintenance costs. Next on the list is centrifugation, which has limited uses as a batch process, but can be very useful in continuous operation with screw decanter centrifuges. Continuous centrifuge operation can be difficult to control and infrastructure is required to convey rather than pump the output. Sedimentation in tailings impoundments achieves high output solids concentrations with time, but is generally preceded by a gravity thickening process to maximise liquor/water recovery and minimise environmental footprint. The gravity thickening process is well established as a means of increasing the solids concentrations of particulate suspensions in a wide range of industries and on a massive scale. Being a continuous process that enables a high throughput, gravity thickening has significant advantages in terms of operating cost and manpower requirements. 
Thickening processes generally feed a flocculated, but otherwise un-networked suspension which is concentrated to a higher solids concentration by gravity. The output solids concentration can be controlled by the throughput (Usher and Scales, 2005). At very high throughputs, the thickener operates as a clarifier with a moderate increase in the underflow solids concentration. At lower throughputs and with the application of raking, higher underflow solids concentrations can be achieved, to a thick upper limit defined by what can be transported through raking and pumping. The challenge in thickening processes is to develop an understanding of how to operate gravity thickeners at high solids throughputs, with high underflow solids concentrations, but with low flocculant use. As described in this paper, a fundamental understanding of the impact of shear processes on dewatering material properties and process performance is being developed.

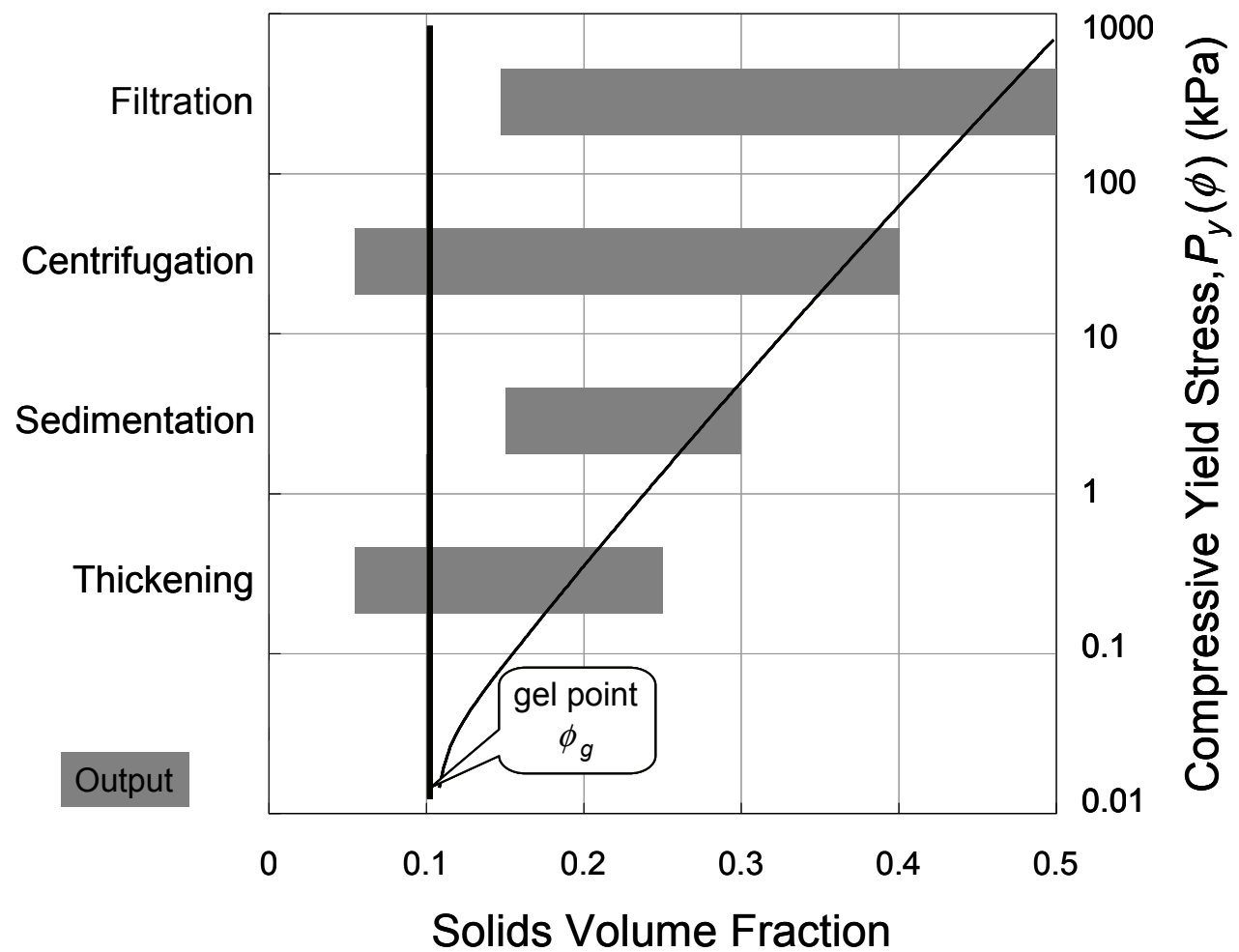

Figure 1 Typical output solids volume fraction ranges for industrial separation equipment, overlaid on a compressive yield stress curve $P_{y}(\phi)$ for a material with a gel point of $10 \% \mathrm{v} / \mathrm{v}$ solids

\subsection{Dewatering theory background}

Dewatering theory developed by Buscall and White (1987) can be applied to determine material properties that describe dewatering behaviour. These material properties describe the rate and extent of dewatering of particulate suspensions as a function of solids concentration. These can then be utilised in numerical computational algorithms to predict dewatering in both industrial and laboratory dewatering processes. The material properties are the compressive yield stress, $P_{y}(\phi)$, and the hindered settling function, $R(\phi)$, with solids concentration defined as the solids volume fraction, $\phi$.

The gel point, $\phi_{g}$, is the minimum solids volume fraction at which a particulate suspension forms a continuous network of particles that can transmit stress. For all solids concentrations above the gel point, the particulate suspension has an inherent network strength, quantified by the compressive yield stress, $P_{y}(\phi)$, in compression and the (shear) yield stress, $\tau_{y}(\phi)$, in shear. The compressive yield stress is defined as the particulate network pressure required to cause a particulate network at a given solids volume fraction, $\phi$, to fail and concentrate to a higher solids volume fraction. The hindered settling function is defined as the hydrodynamic resistance to liquor flow through a particulate suspension as a function of solids volume fraction. It is valid for all solids volume fractions as it can be interpreted as either the movement of liquor past a collection of solids or a particle through a liquid. The hindered settling function can also be viewed as being inversely proportional to the permeability or settling rate of the suspension. 


\subsection{Dewatering characterisation}

Figure 2 shows the $P_{y}(\phi)$ and $R(\phi)$ data and curve fits produced for a typical mineral slurry from analysis of laboratory based dewaterability characterisation tests. These laboratory tests include batch sedimentation and pressure filtration of a flocculated particulate suspension. Equilibrium batch settling tests can be used to determine $P_{y}(\phi)$ near the gel point (Green, 1997; Usher, 2002; de Kretser et al., 2005). Transient batch sedimentation test data can be used to analytically determine $R(\phi)$ from low initial solids concentrations up to near the gel point (Lester et al., 2005; Grassia et al., 2008). Stepped pressure filtration tests are used to determine both $P_{y}(\phi)$ and $R(\phi)$ at high solids concentrations (de Kretser et al., 2001; Usher et al., 2001). Curve fits of the data are produced to interpolate between the low and high solids concentration data, enabling characterisation of materials over the whole range of solids concentrations applicable to thickening.
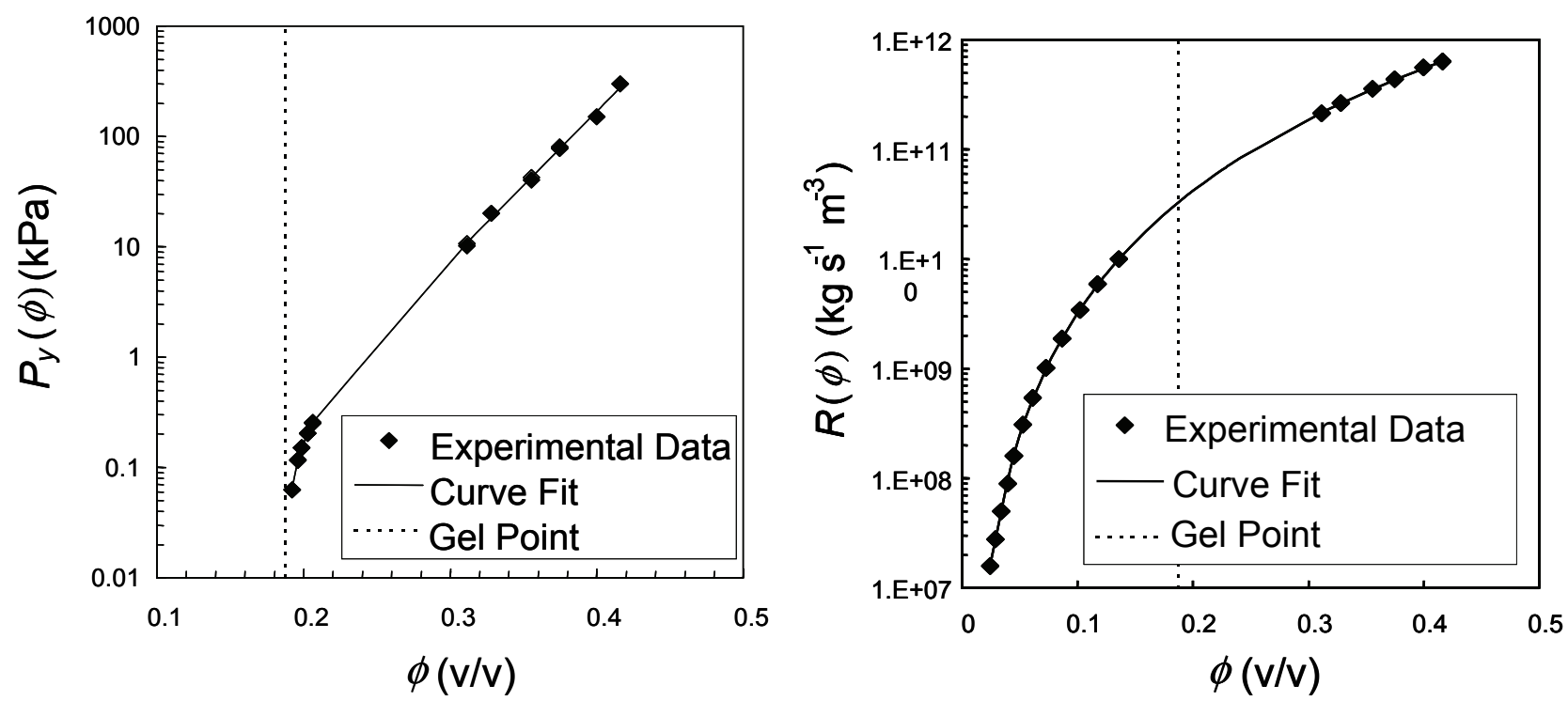

Figure 2 Typical output solids volume fraction ranges for $P_{y}(\phi)$ and $R(\phi)$ data produced with various laboratory dewaterability characterisation tests, overlaid on $P_{y}(\phi)$ and $R(\phi)$ curves

\subsection{Application of dewatering theory}

Dewatering theory has been successful in describing one dimensional dewatering processes such as batch sedimentation (Lester et al., 2005) and piston driven filtration in the laboratory (de Kretser et al., 2001; Usher et al., 2001). On an industrial scale, the dewatering performance of plate and frame filter presses have been accurately predicted (Stickland, 2005; Stickland et al., 2006).

Numerous authors have presented one dimensional differential equations and computational algorithms for predicting transient thickener performance (Bürger et al., 2000b; Lester, 2003; Martin, 2004) and steady state thickener performance (Landman et al., 1988; Landman and White, 1994; Green, 1997; Garrido et al., 2003; Usher and Scales, 2005) from material properties algebraically related to the compressive yield stress and permeability. The outputs of these algorithms enable understanding of how process variations can affect dewatering performance. The key disadvantage of the transient algorithms is that they require large amounts of computation time to model a wide range of conditions. Use of the quicker, simpler steady state thickening algorithms enables a wider range of conditions to be modelled.

As an example of the outputs of these models, the case of a suspension of flocculated red mud from the washer train of an Australian refinery has been investigated (Usher, 2002). The flocculation that occurs in a thickener feedwell can be replicated in the laboratory or pilot plant through plunging in batch sedimentation cylinders, through controlled mixing in a baffled batch reactor or in a continuous pipe reactor. In this case, the input data for the model was measured on-site using samples taken from the underflow and re-flocculated using the same dose of polymer as in the actual device by plunging in a cylinder. Whilst there is a possibility that the material was not flocculated in an identical manner, the difference in predicted versus actual 
performance is substantial. Experimental $P_{y}(\phi)$ and $R(\phi)$ data were obtained on-site using a combination of batch settling tests and filtration. These data were used with the thickener geometry in the algorithm developed by Usher and Scales (2005) to predict steady state dewatering performance.

A range of solids throughput scenarios are presented in Figure 3 for bed heights of 2 and $5 \mathrm{~m}$. At high throughput, usually given as a solids flux (tonnes of solids $/ \mathrm{hr} / \mathrm{m}^{2}$ ), the model predicts that the thickener will give underflow solids that are independent of the operational bed height. As solids flux decreases, the underflow will reach the gel point of the suspension and the dependence on the operational bed height will become more important. The solids concentration in Figure 3 is given as a solids volume fraction, although it is relatively easy to convert to solids weight fraction if the solid and liquid phase densities are known. A closer look at the model data indicates that for underflow solids up to and just in excess of the gel point, the operation of the thickener is limited by permeability alone in that any improvement in the permeability of the settling aggregates will increase the underflow solids concentration. The effect of bed height will be important for solids concentrations above the gel point but the operation will still be limited by permeability unless the solids flux is very low. A survey of thickener operations shows that since the ability to rake and pump the underflow solids is limiting, the majority of operational thickeners will be permeability limited and provided they are run at a bed height in excess of 2-3 m, operational changes that enhance the settling rate of the flocculated solids or the permeability of the solids bed will be beneficial to improving either of underflow solids or solids throughput.

Plotting actual process performance in Figure 3 allows comparison of prediction with reality over the operational bed height range of 2-5 m. In terms of the solids flux through the thickener, the difference was a factor of 16.5. This has been labelled as a performance enhancement (PE) factor in Figure 3. The role of raking and other shear processes in enhancing dewatering has not been accounted for in the modelling. This type of comparison gives an indication of the level to which factors such as shear processes influence the rate and extent of dewatering. Observed performance enhancement factors between 4 and 100 have been reported for red mud thickeners (Usher, 2002; Gladman et al., 2006a; Usher and Scales, 2009), suggesting that the dewatering model generally underestimates thickener throughput at a given underflow solids concentration. Analysis of a broad range of industrial thickeners from the processing of gold ores, red mud, coal tailings, potable water sludge and clay suspensions has produced variable results with typical PE factors varying from 5 to 20 for conventional thickening operations (Gladman et al., 2006b).

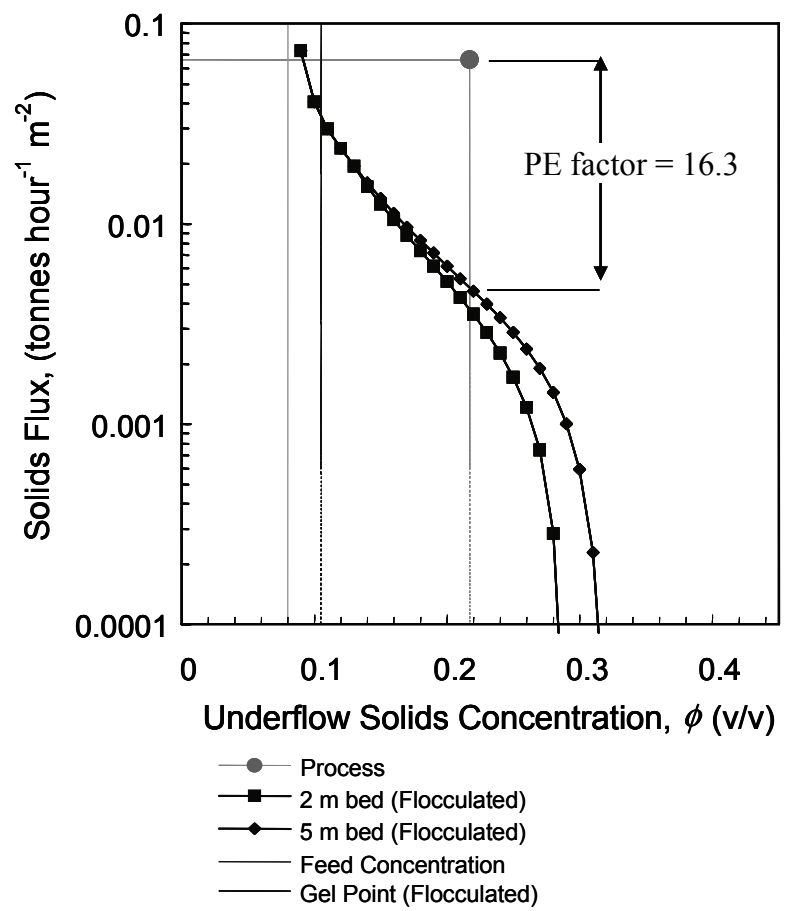

Figure 3 Red mud thickener performance and model prediction of the solids flux versus observed underflow solids volume fraction. The difference between predicted and actual performance is indicated as a performance enhancement (PE) factor (Usher, 2002) 


\section{Shear effects}

The discrepancy between predictions and operational performance for gravity thickeners has driven researchers to investigate the shear related phenomena that cause material properties to change during the thickening process. Origins of the shear forces that are believed to account for these changes include rakes and sloped walls in thickeners. The primary purpose of rakes is to transport material to the underflow discharge point, thus preventing the build-up of a static bed that would otherwise block the thickener. However, it is has been demonstrated that raking can also enhance dewatering performance. Thickener performance is reliant on raking systems and other shear processes to maximise the output solids concentration at high solids throughputs.

It was shown by Channell et al. (2000) that material properties can change in the presence of shear. In addition it has been observed that high shear rates are detrimental to dewatering due to aggregate breakage (Novak and Bandak, 1994; Aziz et al., 2000). For simple batch settling tests, the presence of slow, gentle raking enables improvement in settling behaviour that more detailed analysis shows is due to an increase in the settling rate at all solids concentrations and an increase in the final networked bed solids concentration (Comings et al., 1954; Vesilind and Jones, 1993; Farrow et al., 2000; Johnson et al., 2000; Gladman et al., 2006b; Usher and Scales, 2009). Further, batch settling in conical cylinders has demonstrated that the sloped walls also contribute to an improvement in both the rate and extent of dewatering (Usher and Scales, 2009).

In unpublished work performed within our research group, fluidisation tests have been conducted to mimic the sedimentation zone above the networked bed in a thickener where the suspension is effectively fluidised at a constant solids volume fraction. These fluidisation tests, conducted on flocculated suspensions, have demonstrated that even at low, un-networked solids concentrations, the settling rate can increase with time (Gladman, 2004), largely due to changes in aggregate structure such as densification. At larger times of fluidisation, of the order of hours, the settling rate may then decrease and a growing turbid layer is observed at the sediment-liquid interface, indicative of aggregate breakage. The impact of aggregate densification has been theoretically predicted, using theory by Usher et al. (2009), and used to predict the extent of densification for the observed increases in settling rate. Accompanying aggregate size measurements demonstrate that aggregates do indeed decrease in size over time, but to a much larger extent than predicted by densification theory. The implication here is that both densification and breakage phenomena are difficult to isolate from each other and a useful model needs to account for both simultaneously. Experimental data is required to identify the rates of densification and breakage over a wide range of solids concentrations and shear conditions.

\section{Model development}

Flocculated aggregates, whether formed in a thickener feedwell or in a laboratory test, are assumed to have a fractal structure. Quantitatively, this assumes that a spherical aggregate of radius $\mathrm{R}$, is formed with fractal dimension $D$ such that the mass of solids contained within that aggregate, $m(R)$, is given by:

$$
m(R)=a R^{D},
$$

and the average solids volume fraction within this fractal aggregate, $\phi_{a g g}(R)$, is given by:

$$
\phi_{\text {agg }}(R)=b R^{D-3},
$$

where $a$ and $b$ are constants that can be determined. The implication of a fractal aggregate structure is that the local solids concentration within an aggregate is expected to decrease as the distance from the centre of the aggregate increases. When subjected to shear, these weak outer zones are highly susceptible to deformation through densification and breakage.

Developing a model that accounts for and predicts the impact of changes to aggregate size and structure on the rate and extent of dewatering is demanding. A model has been developed to predict the impact of aggregate densification on dewatering performance (Usher et al., 2009). Building on this, the challenge is to observe and quantitatively describe the impact of aggregate breakage. From there, the influence of shear rate on the rates of densification and breakage need to be quantified. 


\subsection{Aggregate densification}

A depiction of flocculated aggregates with fractal structure is shown in Figure 4A. The overall solids volume fraction of the suspension shown is 0.1 and the pathway for liquid flow is tortuous. With aggregate densification, shown in Figure 4B, the aggregates are smaller and have a more uniform (non-fractal) internal solids concentration distribution. With densification, flow of liquid around the aggregates is much easier with larger pores and channels enabling significantly higher settling velocities. Using the theoretical framework for aggregate densification described by Usher et al. (2009), the impact of aggregate densification on material properties can be theoretically predicted.

The impact of different extents of densification on the hindered settling function, $R(\phi)$, inversely related to the settling velocity, is shown in Figure 5. The change in the hindered settling function is predicted to be an improvement by a factor of order 100 if the aggregate diameter is reduced to $70 \%$ of its original value through densification. Initial experimentation suggests that diameters of between 70 and $80 \%$ of original diameter are attainable.

In addition to settling rate improvements, aggregate densification also impacts the strength of particulate suspensions. The average solids volume fraction within the aggregates, $\phi_{a g g}$, is intrinsically linked to the gel point, $\phi_{g}$. The gel point, physically described as the solids volume fraction at which aggregates form a continuous network structure with packing that is comparable to close packing, can be described by:

$$
\phi_{g}=\varphi_{p} \phi_{\text {agg }},
$$

where $\varphi_{p}$ is the nominal aggregate packing volume fraction at the gel point. When the average solids volume fraction within the aggregates, $\phi_{a g g}$, increases with aggregate densification, the gel point, $\phi_{g}$, logically increases, allowing particulate suspensions to pack to higher solids concentrations. The change in the gel point, $\phi_{g}$, is predicted to increase significantly if the aggregate diameter can be reduced to $70 \%$ of its original value through densification. The related impact is significant the lowering of the compressive yield stress, $P_{y}(\phi)$, as predictions in Figure 6 demonstrate.

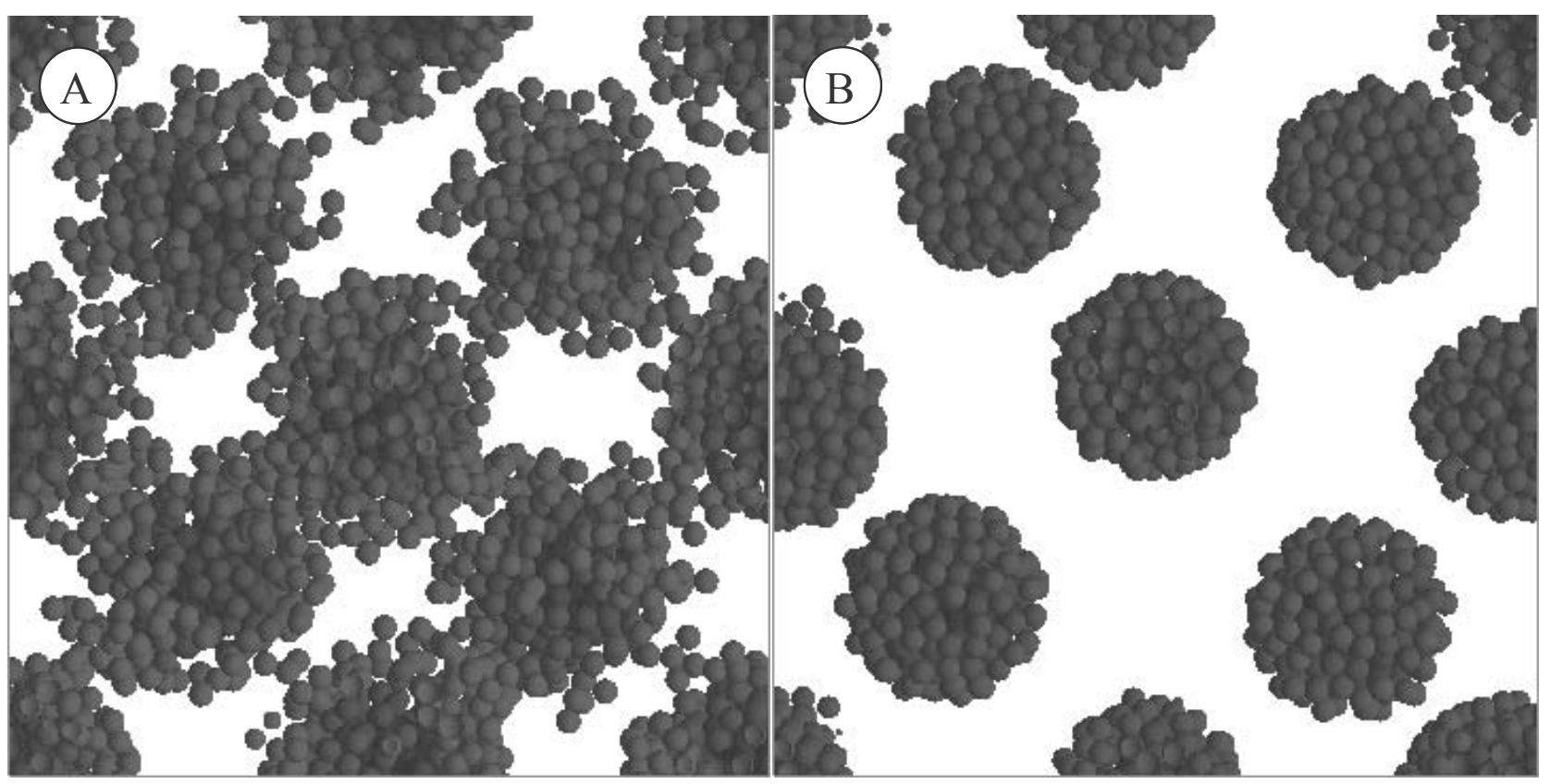

Figure 4 Depiction of flocculated aggregates at an overall solids volume fraction of 0.1. A: with fractal structure before shear and B: after densification as a result of shear 


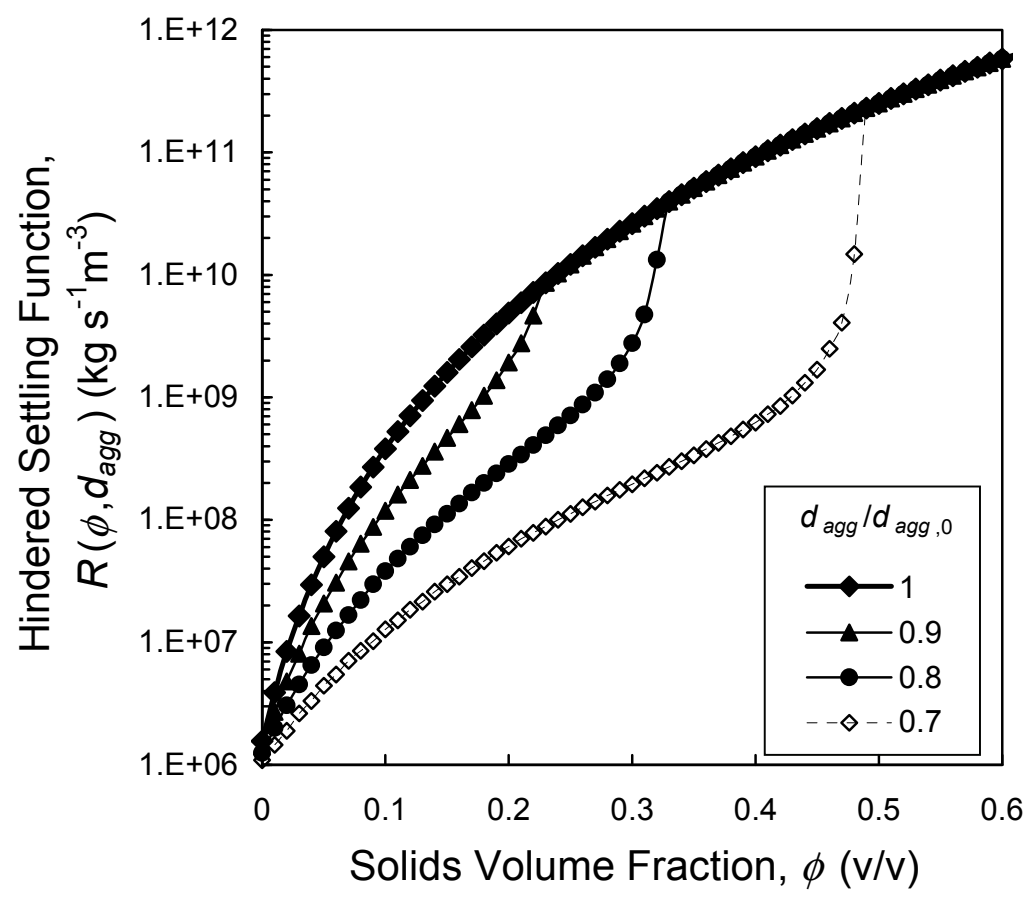

Figure 5 Prediction of hindered settling function for a typical mineral suspension at different extents of densification ranging from undensified, at dagg/dagg, $0=1$, to significant densification at dagg/dagg, $0=0.7$. Predictions based on theoretical framework described by Usher et al. (2009)

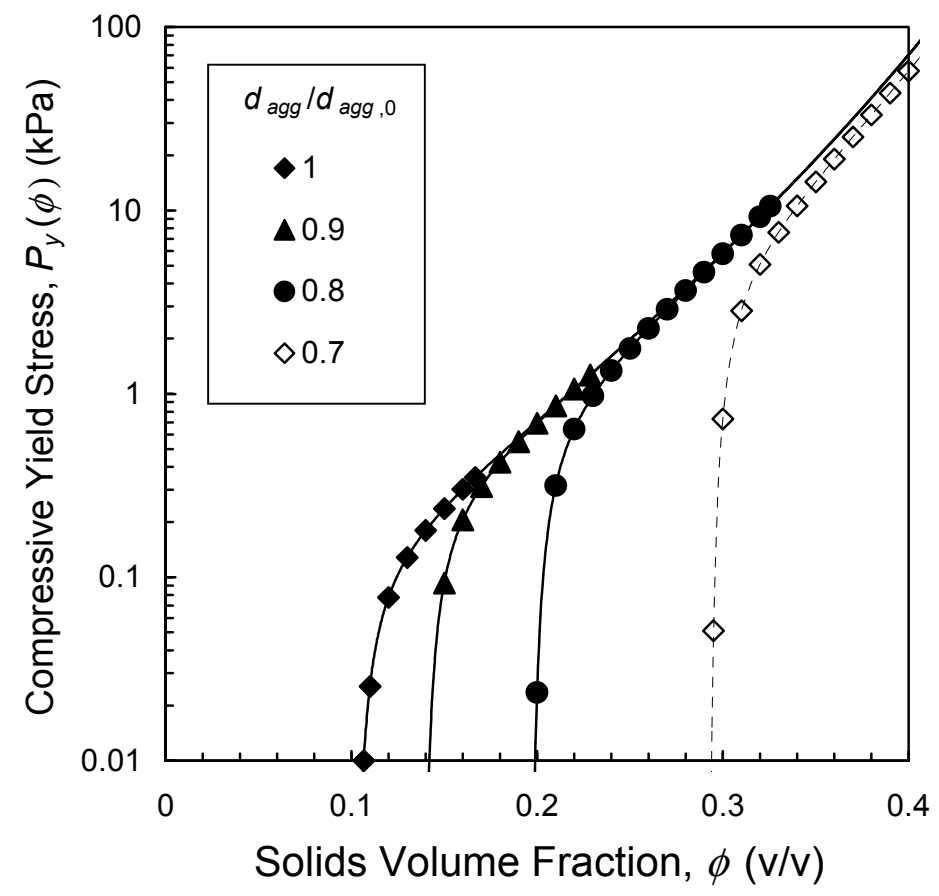

Figure 6 Prediction of compressive yield stress function for a typical mineral suspension at different extents of densification ranging from undensified, at dagg/dagg, $0=1$, to significant densification at dagg/dagg, $0=0.7$. Predictions based on theoretical framework described by Usher et al. (2009) 


\subsection{Aggregate breakage}

As already mentioned, in addition to the beneficial densification phenomena due to shear processes, aggregates are also prone to breakage. The impact of high shear rates and even extended periods of shear at low rates is to cause aggregate breakage. At high shear rates, such as in a feedwell, an aggregate is likely to break into two relatively similar smaller aggregates. However, at the significantly lower shear rates observed in the sedimentation and consolidation zones of a gravity thickener breakage is better described as the erosion of very small aggregates and primary particles from the larger aggregate. The impact of low shear rate breakage or erosion can be visualised in Figure 7. The presence of isolated particles between the aggregates can clog the pathways for liquid flow through the interstices, thus partially negating the benefits of densification.

Quantification of the impact of such a bi-disperse mixture on settling rate can be theoretically performed using polydisperse dewatering models such as those described by Bürger et al. (2000a; 2008), but experimental observations are required to verify that such models can describe such a system where both the aggregates and small particles are ill defined and the aggregates themselves have an inherent porosity that must be accounted for in terms of a mass balance and also in terms of the impact on flow rates.

The impact of breakage on the strength of the network is also complicated, but still theoretically feasible to predict if the size of the eroded particles is assumed to be significantly smaller than the aggregates from which they originate. The impact of breakage itself, through outer particle erosion, is to increase the average solids volume fraction within the fractal aggregate. The overall gel point, $\phi_{g}$, of the mixture can be determined through a material balance.

At low levels of solids fraction broken, the aggregates will form a continuously spanning network and the eroded primary particles will occupy the interstices between the aggregates. With increasing breakage, the primary particles will eventually become networked in the interstices and the aggregates will be isolated in a network of primary particles. The theoretical impact of breakage on the gel point of a typical flocculated mineral slurry can be calculated. The implication here is that even though breakage can hinder liquid flow in the interstices between aggregates, it can have a small positive impact on the gel point.

The observation that shear can increase the extent of dewatering in sedimentation tests (Usher, 2002; Gladman et al., 2005) is consistent with predictions that the gel point will increase with both aggregate densification and breakage. Unfortunately, the implication is that any observed improvement will be due to a combination of both phenomena. Quantifying the effects of densification and breakage in isolation is thus difficult and requires the development of novel experimental design and analysis.
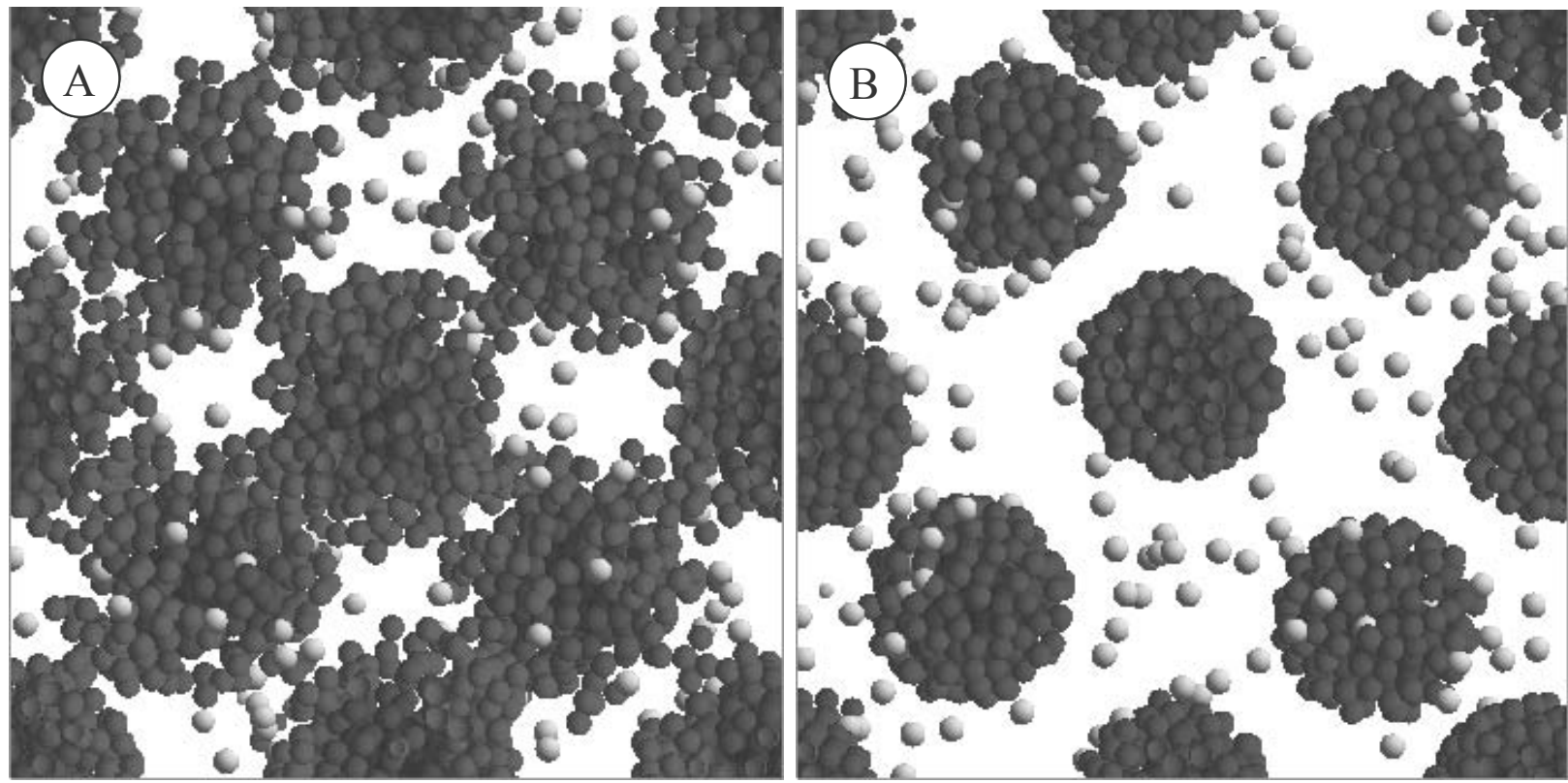

Figure 7 Depiction of isolated particles hindering liquid flow pathways between flocculated aggregates $A$ : with fractal structure and $B$ : after densification 


\section{Conclusions}

Material properties that describe the rate and extent of dewatering of flocculated particulate suspensions can be characterised in the laboratory. These data can be utilised in computational modelling to predict steady state thickener performance. Comparison of these predictions with actual performance has identified that there are phenomena not accounted for in current models. The discrepancy is attributed to shear effects, such as aggregate densification and breakage that change aggregate structure and material properties during the thickening process. A theoretical framework has been developed to enable prediction of the effect of aggregate densification on material properties. Further development of this model to also account for the impact of aggregate breakage is ongoing. Combining such a model with experimental observations and differential equations to describe the rate and extent of densification and breakage will enable significantly improved understanding of how shear can be utilised to optimise thickener performance.

\section{Acknowledgements}

This work was conducted as part of the AMIRA International Limited collaborative research projects P266E and P266F: Improving Thickener Technology and supported by the Australian Research Council (ARC) under the Linkage Scheme. The authors also acknowledge the support of the PFPC (Particulate Fluids Processing Centre), a Special Research Centre of the ARC.

\section{References}

Aziz, A.A.A., de Kretser, R.G., Dixon, D.R. and Scales, P.J. (2000) The Characterisation of Slurry Dewatering, Water Science and Technology, Vol. 41(8), pp. 9-16.

Bürger, R., Concha, F., Fjelde, K.K. and Karlsen, K.H. (2000a) Numerical simulation of the settling of polydisperse suspensions of spheres, Powder Technology, Vol. 113(1-2), pp. 30-54.

Bürger, R., Evje, S., Hvistendahl Karlsen, K. and Lie, K.A. (2000b) Numerical methods for the simulation of the settling of flocculated suspensions, Chemical Engineering Journal, Vol. 80, pp. 91-104.

Bürger, R., García, A., Karlsen, K.H. and Towers, J.D. (2008) A kinematic model of continuous separation and classification of polydisperse suspensions, Computers and Chemical Engineering, Vol. 32(6), pp. 1173-1194.

Buscall, R. and White, L.R. (1987) The Consolidation of Concentrated Suspensions, J. Chem. Soc. Faraday Trans. I 83, pp. 873-891.

Channell, G.M., Miller, K.T. and Zukoski, C.F. (2000) Effects of microstructure on the compressive yield stress, AIChE Journal, Vol. 46(1), pp. 72-78.

Comings, E.W., Pruiss, C.E. and DeBord, C. (1954) Continuous settling and thickening, Ind. Eng. Chem., Vol. 46(6), pp. 1164-1172.

de Kretser, R.G., Usher, S.P. and Scales, P.J. (2005) Comprehensive Dewatering Behaviour Analysis for Fine and Flocculated Materials, American Filtration and Separations Society, 18th Annual Conference, Atlanta GA USA, April 10-13 2005, American Filtration and Separations Society.

de Kretser, R.G., Usher, S.P., Scales, P.J., Boger, D.V. and Landman, K.A. (2001) Rapid Filtration Measurement of Dewatering Design and Optimization Parameters, AIChE J., Vol. 47(8), pp. 1758-1769.

Farrow, J.B., Johnston, R.R.M., Simic, K. and Swift, J.D. (2000) Consolidation and aggregate densification during gravity thickening, Chemical Engineering Journal, Vol. 80(1-3), pp. 141-148.

Garrido, P., Burgos, R., Concha, F. and Bürger, R. (2003) Software for the design and simulation of gravity thickeners, Minerals Engineering, Vol. 16, pp. 85-92.

Gladman, B.R. (2004) The Effect of Shear on Dewatering of Flocculated Suspensions, PhD in Department of Chemical and Biomolecular Engineering, Melbourne, Australia, The University of Melbourne, 332 p.

Gladman, B.R., de Kretser, R.G., Rudman, M. and Scales, P.J. (2005) Effect of shear on particulate suspension dewatering, Trans IChemE, Part A, Chemical Engineering Research and Design, Vol. 83(A7), pp. 933-936.

Gladman, B.R., Usher, S.P. and Scales, P.J. (2006a) Compressive rheology of aggregated particulate suspensions, Korea Australia Rheology Journal, Vol. 18(4), pp. 191-197.

Gladman, B.R., Usher, S.P. and Scales, P.J. (2006b) Understanding the thickening process, in Proceedings of the Ninth International Seminar on Paste and Thickened Tailings, Australian Centre for Geomechanics, Perth, Australia, pp. $5-12$.

Grassia, P., Usher, S.P. and Scales, P.J. (2008) A simplified parameter extraction technique using batch settling data to estimate suspension material properties in dewatering applications, Chem. Eng. Sci., Vol. 63, pp. 1971-1986.

Green, M.D. (1997) Characterisation of Suspensions in Settling and Compression, PhD in Department of Chemical Engineering, Melbourne, Australia, The University of Melbourne, 246 p. 
Johnson, S.B., Scales, P.J., Dixon, D.R. and Pascoe, M. (2000) Use of a superthickener device to concentrate potable water sludge, Water Research, Vol. 34(1), pp. 288-294.

Landman, K.A. and White, L.R. (1994) Solid/Liquid Separation of Flocculated Suspensions, Advances in Colloid and Interface Science, Vol. 51, pp. 175-246.

Landman, K.A., White, L.R. and Buscall, R. (1988) The continuous flow gravity thickener: Steady state behaviour, AIChE J., Vol. 34, pp. 239-252.

Lester, D.R. (2003) Colloidal Suspension Dewatering Analysis, PhD in Department of Chemical and Biomolecular Engineering, Melbourne, Australia, The University of Melbourne, 227 p.

Lester, D.R., Usher, S.P. and Scales, P.J. (2005) Estimation of the Hindered Settling Function $R(\phi)$ from Batch-Settling Tests, AIChE J., Vol. 51(4), pp. 1158-1168.

Martin, A.D. (2004) Optimisation of clarifier-thickeners processing stable suspensions for turn-up/turn-down, Water Research, Vol. 38, pp. 1568-1578.

Novak, J.T. and Bandak, N. (1994) The effect of shear on the dewatering of water treatment residuals, AWWA, Vol. 86(11), pp. 84-91.

Stickland, A.D. (2005) Solid-Liquid Separation in the Water and Wastewater Industries, PhD in Department of Chemical and Biomolecular Engineering, The University of Melbourne, $461 \mathrm{p}$.

Stickland, A.D., de Kretser, R.G., Scales, P.J., Usher, S.P., Hillis, P. and Tillotson, M.R. (2006) Numerical modelling of fixed-cavity plate-and-frame filtration: Formulation, validation and optimisation, Chemical Engineering Science, Vol. 61, pp. 3818-3829.

Usher, S.P. (2002) Suspension Dewatering: Characterisation and Optimisation, $\mathrm{PhD}$ in Department of Chemical Engineering. Melbourne, Australia, The University of Melbourne, $347 \mathrm{p}$.

Usher, S.P., de Kretser, R.G. and Scales, P.J. (2001) Validation of a New Filtration Technique for Dewaterability Characterization, AIChE J., Vol. 47(7), pp. 1561-1570.

Usher, S.P. and Scales, P.J. (2005) Steady state thickener modelling from the compressive yield stress and hindered settling function, Chemical Engineering Journal, Vol. 111(2-3), pp. 253-261.

Usher, S.P. and Scales, P.J. (2009) Predicting settler/clarifier behaviour: The role of shear effects, Filtration, Vol. 9(4), pp. 308-314.

Usher, S.P., Spehar, R. and Scales, P.J. (2009) Theoretical analysis of aggregate densification: Impact on thickener performance, Chemical Engineering Journal, Vol. 151(1-3), pp. 202-208.

Vesilind, P.A. and Jones, G.N. (1993) Channelling in batch thickening, Water Science and Technology, Vol. 28(1), pp. 59-65. 